\title{
HISTOPLASMOSE COMO CAUSA DE PERFURAÇÃO INTESTINAL EM PACIENTE COM SÍNDROME DA IMUNODEFICIÊNCIA ADQUIRIDA
}

\author{
HISTOPLASMOSIS AS CAUSE OF INTESTINAL PERFORATION IN A PATIENT WITH \\ ACQUIRED IMMUNODEFICIENCY SYNDROME
}

\author{
Luiz Alberto Rodrigues de Moraes, TCBC-PA ${ }^{1}$ \\ João Maria Silva Rodrigues ${ }^{2}$ \\ Geraldo Ishak, TCBC-PA ${ }^{3}$ \\ Carlos Onete Coelho Moreira, ACBC-PA ${ }^{4}$
}

\section{INTRODUÇÃO}

A histoplasmose clássica, observada principalmente no continente americano, tem como agente o Histoplasma capsulatum, que penetra no organismo geralmente pelo aparelho respiratório; seus micronídeos são capazes de atravessar os bronquíolos e atingir o parênquima pulmonar; a infecção pode se produzir por via digestiva, ao nível dos intestinos. ${ }^{1,2}$

O $H$. capsulatum é parasita intracelular e sua infecção é controlada pelos linfócitos T Helper, que reconhecem os antígenos e subseqüentemente secretam interferon-gama, que ativa os macrófagos para destruir os parasitas intracelulares; em adição, o histoplasma induz macrófagos a secretarem o fator de necrose tumoral alfa, que estimula outros macrófagos a destruílos. A deficiência de imunidade celular deixa os pacientes mais suscetíveis a infecções disseminadas pelo histoplasma. ${ }^{2}$

Nos casos de histoplasmose disseminada, apenas $10 \%$ se expressam com manifestações gastrointestinais, embora em achados de necrópsia o trato gastrointestinal seja o local mais freqüentemente acometido. ${ }^{1}$

A causa mais freqüente de perfuração intestinal em pacientes portadores de síndrome da imunodeficiência adquirida (SIDA) é reconhecidamente o citomegalovírus, sendo responsável pela maioria dos quadros abdominais agudos nesse grupo..$^{1,3}$
Na literatura foi encontrado o relato somente de três casos de perfuração intestinal em decorrência de histoplasmose, sendo um em paciente com transplante renal ${ }^{4}$, outro num paciente diabético com uso crônico de esteróides ${ }^{5}$ e somente um caso em paciente reconhecidamente com SIDA. ${ }^{1}$

\section{RELATO DO CASO}

MSGF, 34 anos, sexo feminino, marido faleceu vítima de SIDA. Foi internada no Hospital Universitário João de Barros Barreto, dia 3/12/96, com teste anti-HIV positivo, febre, perda ponderal de $10 \mathrm{~kg}$ em um mês, diarréia, com fezes pastosas, tosse produtiva, expectoração amarelada, apresentando à ausculta pulmonar murmúrio vesicular rude, roncos, estertores finos e grossos. Iniciou-se antibioticoterapia, sintomáticos, associados a epivir, saquinavir, AZT. Os exames de pesquisa de BAAR e fungos no escarro foram negativos. Ao hemograma apresentou pancitopenia, à esofagogastroduodenoscopia observou-se moniliase esofageana leve, em esôfago proximal.

No dia 25/12 sentiu dor em baixo ventre, que evoluiu com distensão abdominal, timpanismo, descompressão brusca positiva nos quatro quadrantes abdominais, ruídos hidroaéreos presentes e diminuídos. Radiografia panorâmica do abdome: imagens sugestivas de níveis hidroaéreos, distensão de alça.

1. Chefe do Serviço de Cirurgia Geral do HUJBB-UFPa.

2. Interno do Serviço de Cirurgia Geral do HUJBB-UFPa. Monitor da disciplina Gastroenterologia-UFPa.

3. Staff do Serviço de Cirurgia Geral do HUJBB-UfPa. Auxiliar de ensino de Clínica Cirúrgica - Universidade do Estado do Pará.

4. Staff do Serviço de Cirurgia Geral do HUJBB-UFPa.

Recebido em 17/6/97

Aceito para publicação em 26/1/98

Trabalho realizado no Serviço de Cirurgia Geral do Hospital Universitário João de Barros Barreto - Universidade Federal do Pará (HUJBB-UFPa). 
Dia 28/12, permanecendo o quadro de dor abdominal intensa, ruídos hidroaéreos negativos, na radiografia abdominal evidenciavam-se níveis hidroaéreos, pneumoperitônio, alça intestinal distendida com edema de parede. Foi submetida à laparotomia exploradora, onde os achados foram grande quantidade de secreção purulenta, duas perfurações em íleo, sendo a primeira a cerca de $30 \mathrm{~cm}$ da válvula íleo-cecal e a segunda a $40 \mathrm{~cm}$ A conduta cirúrgica foi enterectomia segmentar e ileostomia. Ao exame histopatológico da peça operatória (segmento ileal) o diagnóstico foi histoplasmose intestinal, enterite aguda.

A paciente evoluiu com septicemia e falência de múltiplos órgãos e sistemas, vindo a óbito dia 05/01/1997 no oitavo dia de pós-operatório.

\section{DISCUSSÃO}

Paciente com história clínica epidemiológica e exames laboratoriais positivos para SIDA, apresentando quadro clínico de abdome agudo perfurativo, tendo sido levada à cirurgia com a hipótese diagnóstica de perfuração por citomegalovírus, que, de acordo com a literatura, ${ }^{1,3}$ é a causa mais freqüente de perfuração intestinal em pacientes com SIDA.

A conduta cirúrgica tomada foi a preconizada pela literatura, que advoga a realização de estomias associada à ressecção do segmento comprometido. ${ }^{3}$

O exame histopatológico demonstrou claramente o histoplasma como responsável pelas perfurações, o que representa uma raridade, visto que este seria o quarto caso relatado pela literatura, ${ }^{1,4,5}$ sendo que apenas este caso e outro descrito por Heneghan ${ }^{1}$ foram em pacientes com SIDA.

A paciente veio a óbito no oitavo dia de pós-operatório, em quadro de insuficiência de múltiplos órgãos e sistemas, o que é de acordo com a literatura. ${ }^{3}$

Chama-se atenção para a possibilidade de ao manusearse pacientes com SIDA, além da perfuração por citomegalovírus, devemos ter em mente a histoplasmose como causa de abdome agudo.

\begin{abstract}
Thirty-four years old patient, female, husband died of AIDS (Acquired Immunodeficiency Sindrome). She's confined to Hospital Universitário João de Barros Barreto, with a positive test for AIDS, fever, $10 \mathrm{~kg} / \mathrm{month}$ of weight loss, diarrhea with gummy faeces, productive cough, yellowish sputum. The therapy was initiated, symptomatic, associated to Epivir, Saquinavir and AZT. The searching exams for Alcohol Ácid Resistant Bacili and fungi in the sputum were negative. At the hemogram was shown a pancytopenia, the esophagogastroduodenunscopy showed light esophago moniliasis. During commitment presented a perforating acute abdomen chart, the abdominal radiography showed hidroair levels, pneumoperitoneum, enlarced bowels with swollen wall. She was undertaken a surgery with diagnosis hypothesis of cytomegalovirus perforation, which accordin to literature is the most frequent cause of intestinal perforation in patients with AIDS.
\end{abstract}

Key Words: AIDS; Histoplasmosis; Intestinal histoplasmosis; Intestinal perforation; Ileal perforation.

\section{REFERÊNCIAS}

1. Heneghan SJ, Li J, Petrossian E, et al - Intestinal perforation from gastrointestinal histoplasmosis in acquiride immunodeficiency syndrome. Arch Surg 1993; 128: 464-466.

2. Samuelson J, Lichtenberg FV - Infectious diseases. In: Cotran RS, Robbins SL, Kumar V - Pathologic basis of disease, 5th Edition, USA, Ed. W.B. Saunders Company, 1994: 327-328.

3. Steinman M, Steinman E, Poggetti RS, et al - Urgências cirúrgicas abdominais em pacientes com síndrome da imunodeficiência adquirida. Rev Ass Med 1996; 42:1.19-24.

4. Brett MJ, Kwan JT, Bending MR - Caecal perforation in a renal transplant patient with disseminated histoplasmosis. J Clin Pathol 1988;41: 992-995.
5. Lee SH, Barnes WG, Hodges GR, et al - Perforated granulomatous colitis caused by histoplasma capsulatum. Dis Colon Rectum 1985; 28: $171-176$.

\section{ENDEREÇO PARA CORRESPONDÊNCIA}

Dr. Luiz Alberto Rodrigues Moraes

Trav. Benjamin Constant, 1520

660150-060 - Belém - PA 